\title{
Mapping momentum-dependent electron-phonon coupling and non-equilibrium phonon dynamics with ultrafast elec- tron diffuse scattering
}

\author{
Mark J. Stern ${ }^{1}$, Laurent P. René de Cotret $^{1}$, Martin R. Otto ${ }^{1}$, Robert P. Chatelain ${ }^{1}$, Jean- \\ Philippe Boisvert ${ }^{1}$, Mark Sutton ${ }^{1}$, and Bradley J. Siwick ${ }^{1,2, *}$ \\ ${ }^{1}$ Department of Physics, Center for the Physics of Materials, McGill University, 3600 University Street, \\ Montreal, Quebec, Canada H3A 2T8 \\ ${ }^{2}$ Department of Chemistry, McGill University, 801 Sherbrooke Street W, Montreal, Quebec, Canada \\ H3A 0B8
}

\begin{abstract}
We show that ultrafast electron diffuse (inelastic) scattering provides a detailed, momentum-resolved view of electron-phonon and phonon-phonon coupling across the entire Brillouin zone. Ultrafast time-resolution and scattering selection rules can distinguish between phonon branches without energyresolution.
\end{abstract}

\section{Introduction}

The nature of the couplings within and between lattice and charge degrees of freedom is central to condensed matter and materials physics. These interactions are determining to phenomena as diverse as superconductivity, charge density waves and carrier mobility in semiconductors and metals[1]. Despite their fundamental role, detailed momentum-dependent information on the strength of electron-phonon and phonon-phonon coupling (EPC and PPC, respectively) across the entire Brillouin zone (BZ) has proved elusive. Ultrafast electron diffuse scattering (UEDS) provides the answer. The information UEDS provides is analogous to time-resolved, angle resolved photoelectron spectroscopy (TR-ARPES) but for the phonon system rather than the electron system. That is, UEDS provides a measure of momentumdependent phonon population dynamics in the way that TR-ARPES provides a measure of the momentum-dependent occupation dynamics of electronic states[2].

We show that this technique is particularly well suited as a probe of $2 \mathrm{D}$ materials using thin graphite as a model system. Specifically, optical excitation at $800 \mathrm{~nm}$ with $35 \mathrm{fs}$ laser pulses drives vertical electronic transitions of a $\pi-\pi^{\star}$ character on the well-known Dirac cones[3] of single crystal graphite samples. This excitation impulsively photo-dopes the material with a non-equilibrium electron-hole plasma with carrier density controllable by excitation fluence. In this work we use UEDS patterns to determine how the energy stored in the hot electron system couples to the phonons and how the phonon system subsequently thermalizes.

\footnotetext{
*e-mail: bradley.siwick@mcgill.ca
} 

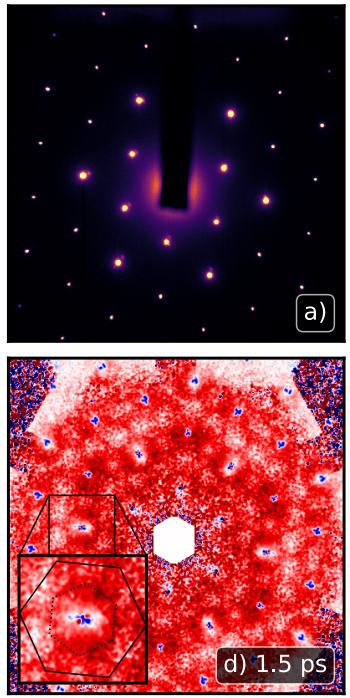
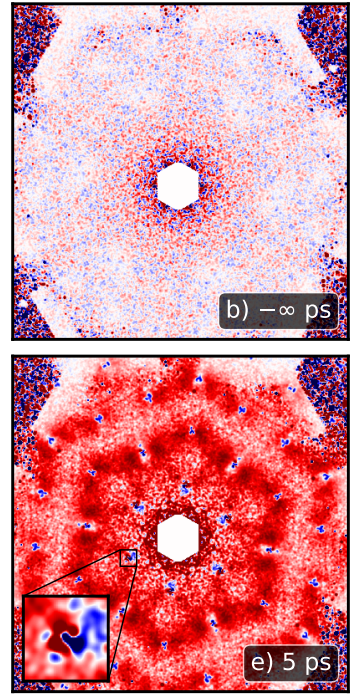

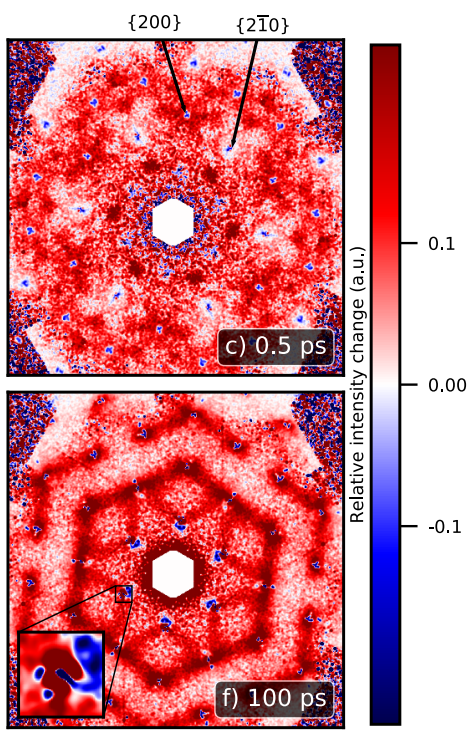

Figure 1. Evolution of $\Delta I(\boldsymbol{q}, \tau) /|\boldsymbol{q}|^{2}=\frac{1}{|\boldsymbol{q}|^{2}} \frac{I(\boldsymbol{q}, \tau)-I(\boldsymbol{q},-\infty)}{I(\boldsymbol{q},-\infty)}$ following photo-excitation of graphite (35 fs, $800 \mathrm{~nm}, 12 \mathrm{~mJ} / \mathrm{cm}^{2}$ ). The dramatic changes reflect the non-equilibrium phonon populations and their time dependence. a) Raw diffraction pattern of graphite along the [001] zone axis. b) Differential scattering flat-field $\Delta I(\boldsymbol{q}, \tau)$ at a time before optical excitation (indicating signal-to-noise and artefactual edges). c) $\Delta I(\boldsymbol{q}, 0.5 \mathrm{ps})$ provides a map of the relative strength of the $\boldsymbol{q}$-dependent EPC coupling through the increased occupancy of strongly-coupled modes. d) Non-equilibrium phonon dynamics: relaxation of the transient population of strongly-coupled modes into mid-Brillouin zone (inset) acoustic modes e) - f) subsequent thermalization.

\section{Results and discussion}

UEDS patterns provide an ultrafast snapshot of the change in scattered intensity, $\Delta I(\boldsymbol{q})$, for all scattering vectors, $\boldsymbol{q} . \Delta I(\boldsymbol{q})$ is proportional to a sum over the instantaneous occupancy of all phonon branches, $j$, multiplied by the norm of the so-called one-phonon structure factor[4], $F_{j}(\boldsymbol{q})$. By calculating the one-phonon structure factor via density functional perturbation theory, one can identify extinctions in $F_{j}(\boldsymbol{q})$ at particular points of the diffuse scattering pattern for a given phonon mode. Depending on the chosen $\boldsymbol{q}$, several of the phonon modes can be inactive $\left(F_{j}(\boldsymbol{q}) \approx 0\right)$, reducing the number of phonon branches that can contribute at that point. If only one mode contributes than its individual population dynamics can be observed directly even without energy resolution.

Here, we show that UEDS patterns of photo-excited graphite (Fig. 1) map the relative EPC and PPC strength through their profound sensitivity to photoinduced changes in phonon populations. Earlier work suggested two strongly-coupled optical modes, $A 1^{\prime}$ and $E_{2 g}$ as the initial reservoir into which the electronic excitation energy flows[5]. Diffuse scattering peaks located at the BZ corners ( $K$-points) in Fig. 1 c) and Fig. 2 b) are the first direct evidence of this strong electron phonon coupling to $A 1^{\prime}$. In addition, Fig. $1 \mathrm{c}$ ) indicates that coupling is strong for the entire longitudinal optical (LO) branch between $\Gamma-K$ not only for $\Gamma-E_{2 g}$ mode, as previously reported.

On longer time-scales the dynamics evident in the phonon system remain rich. The diffuse scattering patterns in Fig. $1 \mathrm{~d}-\mathrm{f}$ ) reveal the decay channels for the generated population of strongly-coupled optical phonons as they relax. The time-scales separation between the 


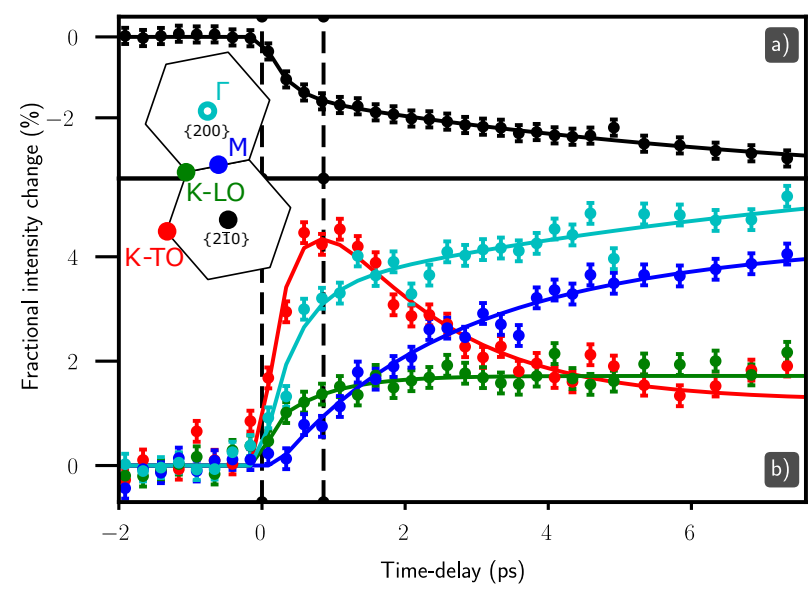

Figure 2. Ultrafast electron diffuse scattering a) Intensity of the (21̄0) Bragg peak showing profoundly non-exponential Debye-Waller dynamics. b) $\Delta I(\boldsymbol{q}, \tau)$ at select points in the BZ (inset) shows rich dynamics that provide detailed information on the instantaneous phonon occupancies when combined with phonon scattering selection rules.

EPC into $K-A_{1}^{\prime}$ and $\Gamma-E_{2 g}(200-400$ fs $)$ and the subsequent decay out of these modes $(1-3 \mathrm{ps})$ means that the diffuse scattering pattern at $1.5 \mathrm{ps}$ maps the momentum-dependent decay probability of the optical modes in a manner analogous to the way in which the $0.5 \mathrm{ps}$ pattern indicates their relative EPC strength. The hexagonal distribution of diffuse intensity approximately halfway between $\Gamma$ and the BZ edges, Fig. 1d) (inset), is associated with the optical phonon to acoustic phonon decay channels. At even later times, a subsequent relaxation channel is evident in the increase in the diffuse halos surrounding the Bragg peaks in Fig. 1 e) and f) (inset).

UEDS provides direct, momentum resolved measurements of the relative strength of EPC and PPC in graphite through the technique's ability to follow phonon population dynamics with femtosecond time resolution. UEDS is also profoundly complementary to ultrafast TRARPES. Together these methods can provide a complete picture of the dynamics within and between electron and phonon sub-systems, and help unravel the physics of complex phases where the intertwined nature the electron-lattice systems determine material properties.

\section{References}

[1] Grimvall, G., The electron-phonon interaction in metals., (North-Holland Pub. Co., 1981).

[2] I. Gierz, F. Calegari, S. Aeschlimann, M. Chávez Cervantes, C. Cacho, R. T. Chapman, E. Springate, S. Link, U. Starke, C. R. Ast, A. Cavalleri, "Tracking Primary Thermalization Events in Graphene with Photoemission at Extreme Time Scales", Phys. Rev. Lett. 115(8), 86803 (2015).

[3] A. H. Castro Neto, F. Guinea, N. M. R. Peres, K. S. Novoselov, A. K. Geim, "The electronic properties of graphene.", Rev. Mod. Phys. 81(1), 109-162 (2009).

[4] R. Xu, T. C. Chiang, "Determination of phonon dispersion relations by X-ray thermal diffuse scattering.", Z. Kristallogr. 220, 1009-1016 (2005).

[5] T. Kampfrath, L. Perfetti, F. Schapper, C. Frischkorn, M. Wolf, "Strongly Coupled Optical Phonons in the Ultrafast Dynamics of the Electronic Energy and Current Relaxation in Graphite.", Phys. Rev. Lett. 95(18), 187403 (2005). 